\title{
ON TOPOLOGIES FOR FUNCTION SPACES
}

\author{
RALPH H. FOX
}

Given topological spaces $X, T$, and $Y$ and a function $h$ from $X \times T$ to $Y$ which is continuous in $x$ for each fixed $t$, there is associated with $h$ a function $h^{*}$ from $T$ to $F=Y^{x}$, the space whose elements are the continuous functions from $X$ to $Y$. The function $h^{*}$ is defined as follows: $h^{*}(t)=h_{t}$, where $h_{t}(x)=h(x, t)$ for every $x$ in $X$. The correspondence between $h$ and $h^{*}$ is obviously one-to-one.

Although the continuity of any particular $h$ depends only on the given topological spaces $X, T$, and $Y$, the topology of the function space $F$ is involved in the continuity of $h^{*}$. It would be desirable to so topologize $F$ that the functions $h^{*}$ which are continuous are precisely those which correspond to continuous functions $h$. It has been known for a long time that this is possible if $X$ satisfies certain conditions, chief among which is the condition of local compactness (Theorem 1). This condition is often felt to be too restrictive (since it practically excludes the possibility of $X$ itself being a function space), and several years ago, in a letter, Hurewicz proposed to me the problem of defining such a topology for $F$ when $X$ is not locally compact. At that time I showed by an example (essentially Theorem 3) that this is not generally possible. Recently I discovered that, by restricting the range of $T$ in a very reasonable way, one of the standard topologies for $F$ has the desired property even for spaces $X$ which are not locally compact (Theorem 2). In this last result the condition of local compactness is replaced by the first countability axiom and this appeals to me as a less troublesome condition.

It should be pointed out that the problem is motivated by the special case in which $T$ is the unit interval. When $T$ is the unit interval, $h$ is a homotopy and $h^{*}$ is a path in the function space; in the topology of deformations, equivalence of the concepts of "homotopy" and of "function-space path" is usually required.

Among the various possible topologies for $F$ there is one, which I shall call the compact-open ${ }^{1}$ (co.o.) topology, which seems to be the most natural. For any two sets, $A$ in $X$ and $W$ in $Y$, let $M(A, W)$ denote the set of mappings $f \in F$ for which $f(A) \subset W$. The co.o. topology is defined by selecting as a sub-basis for the open sets of $F$ the

Presented to the Society, November 25, 1944; received by the editors January 2, 194.5.

1 Terminology followed in this note is generally that of Lefschetz, Algebraic topology, Amer. Math. Soc. Colloquium Publications, vol. 27, New York, 1942. 
sets $M(A, W)$ where $A$ ranges over the compact subsets of $X$ and $W$ ranges over the open subsets of $Y$.

THEOREM 1. If $X$ is regular and locally compact, $Y$ an arbitrary topological space, and if $F$ has the co.o. topology, then continuity of $h$ is equivalent to continuity of $h^{*}$ for any topological space $T$.

THEOREM 2. If $X$ is a space which satisfies the first countability axiom, $Y$ an arbitrary topological space, and if $F$ has the co.o. topology, then continuity of $h$ is equivalent to continuity of $h^{*}$ for any $T$ which satisfies the first countability axiom.

THEOREM 3. If $X$ is separable metrizable and $Y$ is the real line, then in order that it be possible to so topologize $F$ that continuity of $h$ and of $h^{*}$ are equivalent, it is necessary and sufficient that $X$ be locally compact.

Lemma 1. If $F$ has the co.o. topology, then continuity of $h$ implies continuity of $h^{*}$ under no restrictions on the topological spaces $X, T$, and $Y$.

Proof. Let $W$ be an open set in $Y$ and $A$ a compact set in $X$ and let $t_{0}$ be a point in $h^{*-1}(M(A, W))$. Then $A \times t_{0} \subset h^{-1}(W)$. Since $h^{-1}(W)$ is open it is the union of open sets $U_{\alpha} \times V_{\alpha}$. Since $A$ is compact, $A \times t_{0}$ is contained in a finite union $U_{i=1}^{n} U_{i} \times V_{i}$ with each $V_{i}$ a neighborhood of $t_{0}$. Then $\bigcap_{i=1}^{n} V_{i}$ is an open neighborhood of $t_{0}$ and is contained in $h^{*-1}(M(A, W))$.

Proof of Theorem 1. In view of the lemma it is sufficient to prove that continuity of $h^{*}$ implies continuity of $h$. Let $W$ be an open set in $Y$ and let $\left(x_{0}, t_{0}\right)$ be a point in $h^{-1}(W)$. Since $h^{*}\left(t_{0}\right)$ is continuous in $x$ there exists an open neighborhood $U$ of $x_{0}$ such that $h^{*}\left(t_{0}\right) \in M(U, W)$. Because of the conditions on $X$ there is an open neighborhood $R$ of $x_{0}$ such that $\bar{R}$ is compact and contained in $U$. Since $M(\bar{R}, W)$ is open and contains $h^{*}\left(t_{0}\right)$ there is an open neighborhood $V$ of $t_{0}$ such that $h^{*}(V) \subset M(\bar{R}, W) \subset M(R, W)$. Thus $R \times V$ is an open neighborhood of $\left(x_{0}, t_{0}\right)$ which is contained in $h^{-1}(W)$.

Proof of Theorem 2. As before we have to prove that continuity of $h^{*}$ implies continuity of $h$. Let $W$ be an open set in $Y$ and suppose that $h^{-1}(W)$ is not open. Then there is a point $\left(x_{0}, t_{\theta}\right)$ in $h^{-1}(W)$ which is also in the closure of the complement of $h^{-1}(W)$. Let $\left\{G_{n}\right\}$ be a base for the open sets of $X \times T$ at the point $\left(x_{0}, t_{0}\right)$ and choose, for each integer $n$, a point $\left(x_{n}, t_{n}\right)$ in the intersection of $\bigcap_{i \geqq n} G_{i}$ and the complement of $h^{-1}(W)$. Since $h^{*}\left(t_{0}\right)$ is continuous in $x$ there exists an open neighborhood $U$ of $x_{0}$ such that $h^{*}\left(t_{0}\right) \in M(U, W)$. Let 
$A=U \cap \bigcup_{n-0}^{\infty} x_{n}$. Since $A$ is compact, $M(A, W)$ is open and since $h^{*}$ is continuous and $t_{0} \in h^{*-1}(M(A, W))$, there is a neighborhood $V$ of $t_{0}$ such that $h^{*}(V) \subset M(A, W)$. There is an integer $N$ such that $x_{n} \in U$ and $t_{n} \in V$ whenever $n>N$. Hence $h\left(x_{n}, t_{n}\right) \in W$ for every $n$ greater than $N$. This contradiction with the choice of the points $\left(x_{n}, t_{n}\right)$ proves that $h^{-1}(W)$ is open. Thus $h$ is continuous.

LEMMA 2. Let $X$ be a separable metrizable space, let $Y$ be the real line, and suppose that the topology of $F$ is such that continuity of $h$ for $T=[0,1]$ implies the continuity of $h^{*}$. Let $W=(a, b)$ be a finite open interval in $Y$ and let $A$ be a closed subset of $X$ which is not compact. Then the set $M(A, W)$ has no interior points.

Proof. Since $A$ is not compact there is a sequence $\left\{x_{n}\right\}$ in $A$ such that $\cup_{n=1}^{\infty} x_{n}$ is closed in $X$. Given any element $h^{*}(0)$ of the set $M(A, W)$ let us define

$$
h_{t}\left(x_{n}\right)=\min \left\{1+b, h_{0}\left(x_{n}\right)+n t\right\} .
$$

Since the function $h$ is defined over the closed set $X \times[0] \cup\left(\cup_{n-1}^{\infty} x_{n}\right)$ $\times[0,1]$ it may be extended continuously over the normal space $X \times[0,1]$. If $t>0$ there is an integer $n$ such that $a+n t>1+b$; hence $h^{*}(t)$ is in the complement of $M(A, W)$ for every positive $t$. By hypothesis the topology of $F$ is such that $h^{*}$ is continuous. Hence $h^{*}(0)$ belongs to the closure of the complement of $M(A, W)$.

LEMMA 3. If the topology for $F$ is such that continuity of $h^{*}$ always implies continuity of $h$ then, given a point $x_{0}$ in $X$, an open set $W$ in $Y$, and an element $f_{0}$ in $M\left(x_{0}, W\right)$, there is a neighborhood $R$ of $x_{0}$ such that $M(R, W)$ is a neighborhood of $f_{0}$ in $F$.

Proof. Define $\phi(x, f)=f(x)$ for every $(x, f) \in X \times F$. Since $\phi^{*}(f)=f$, $\phi^{*}$ is continuous and hence $\phi$ is also continuous. Since $\phi^{-1}(W)$ is therefore open there must be a neighborhood $R$ of $x_{0}$ and a neighborhood $V$ of $f_{0}$ such that $\phi(R, V) \subset W$. Thus $f_{0} \in V \subset M(R, W)$ and hence $M(R, W)$ is a neighborhood of $f_{0}$ in $F$.

Proof of Theorem 3. Let $W$ be the finite open interval $(a, b)$ and suppose that the topology of $F$ is such that continuity of $h$ and of $h^{*}$ are equivalent for every $T$. From Lemma 3 it follows that, given any point $x_{0}$ in $X$ and any element $f_{0}$ in $M\left(x_{0}, W\right)$, there is a neighborhood $R$ of $x_{0}$ such that $M(R, W)$ is a neighborhood of $f_{0}$ in $F$. Since $X$ is regular there is a neighborhood $U$ of $x_{0}$ whose closure is contained in $R$, so that $M(\bar{U}, W)$ is also a neighborhood of $f_{0}$. Since $f_{0}$ is an interior point of $M(\bar{U}, W)$ it follows from Lemma 2 that $\bar{U}$ is com- 
pact. Thus $X$ must be locally compact. This proves the necessity of the condition; sufficiency is a consequence of Theorem 1.

CoRollary. If $Y$ is the real line and $X$ is separable metrizable but not locally compact, then $F$ does not satisfy the first countability axiom in the co.o. topology.

Proof. Let $W$ be the finite open interval $(a, b)$. If $F$ satisfied the first countability axiom then Theorem 2 would apply to yield the continuity of the function $\phi$ defined above. If $x_{0}$ is a point at which $X$ is not locally compact and $f_{0}$ any element in $M\left(x_{0}, W\right)$, then it follows from the proof of Lemma 3 that there is a neighborhood $R$ of $x_{0}$ such that $M(R, W)$ is a neighborhood of $f_{0}$ in $F$. Let $U$ be a neighborhood of $x_{0}$ whose closure is contained in $R$, so that $f_{0}$ is an interior point of $M(\bar{U}, W)$. Since $\bar{U}$ is not compact this is not in agreement with Lemmas 1 and 2. This contradiction shows that $F$ does not satisfy the first countability axiom in the co.o. topology.

SYRACUSE UNIVERSITY 